\title{
CINÉTICA DE EVOLUÇÃO DE DIÓXIDO DE CARBONO EM ÁREA DE CAATINGA EM SÃO JOÃO DO CARIRI-PB ${ }^{1}$
}

\author{
Kallianna Dantas Araujo², Renilson Targino Dantas³, Albericio Pereira de Andrade ${ }^{4}$ e Henrique Nunes
} Parente $^{5}$

\begin{abstract}
RESUMO - Em decorrência da importância dos elementos meteorológicos nos diversos processos que ocorrem no solo, objetivou-se avaliar a cinética de evolução do dióxido de carbono (atividade microbiana) associada aos elementos meteorológicos em áreas de Caatinga, nas condições do semiárido paraibano. O experimento foi instalado em campo, na área da Bacia Escola/CCA/UFPB, Município de São João do Cariri, PB. A atividade microbiana diurna, medida pela liberação de $\mathrm{CO}_{2}$, foi avaliada mensalmente durante o período de janeiro a dezembro de 2008, em três áreas contíguas de Caatinga, correspondendo aos tratamentos: $\mathrm{T}_{1}\left(10\right.$ animais -1 animal/3.200 $\left.\mathrm{m}^{2}\right), \mathrm{T}_{2}(5$ animais -1 animal/6.400 $\mathrm{m}^{2}$ ) e $\mathrm{T}_{3}$ (Controle - Sem animais). A área do experimento totalizou 9,6 ha, e em cada tratamento foi utilizada uma área de 3,2 ha. Foram selecionados em cada tratamento seis pontos de coleta (definidos ao acaso). Os tratamentos foram distribuídos em delineamento de blocos inteiramente casualizados e arranjados em parcela subsubdividida (3x12x6), sendo três taxas de lotação (Parcela), 12 épocas de avaliação (Subparcelas) e 6 h de avaliação (Subsubparcelas) e três blocos. A metodologia baseou-se no princípio de que o $\mathrm{CO}_{2}$ liberado por uma área de solo é absorvido por uma solução de $\mathrm{KOH}$ 0,5 N e determinado por titulometria com $\mathrm{HCl}$ 0,1 N. A evolução do $\mathrm{CO}_{2}$ variou ao longo do dia em função dos elementos meteorológicos, principalmente temperatura. A taxa de $\mathrm{CO}_{2}$ apresentou tendência à maior liberação no final da tarde. Em decorrência da importância da temperatura do solo e do ar nos inúmeros processos que ocorrem no solo, são necessários mais estudos na região semiárida do Brasil.
\end{abstract}

Palavras-chave: Semiárido, Clima e Atividade microbiana.

\section{KINETICS OF CARBON DIOXIDE EVOLUTION IN AN AREA OF CAATINGA IN SÃO JOÃO DO CARIRI, PB}

\begin{abstract}
Because of the importance of meteorological variables in different processes that occur in the soil, the objective of this work was to evaluate the kinetics of carbon dioxide evolution (microbial activity) associated with the meteorological elements in areas in caatinga in the semiarid conditions of Paraiba. The experiment was set in the field, in the Bacia Escola/CCA/UFPB, municipality of São João do Cariri - PB. The daytime microbial activity, measured by the release of $\mathrm{CO}_{2}$, was evaluated monthly from January to December 2008, in three adjacent areas of Caatinga corresponding to the treatments: $T_{1}$ (10 animals -1 animal/3,200 $\mathrm{m}^{2}$ ), $T_{2}$ (5 animals - 1 animal/6,400 $\mathrm{m}^{2}$ ) and $T_{3}$ (control - no animals). The total area of the experiment was 9.6 ha and for each treatment it was used an area of 3.2 ha. Six points of collection (set at random)were selected from each treatment. The treatments were distributed in completely randomized block design arranged in sub-divided plots $(3 \times 12 \times 6)$, and 3 stocking rates (Plot), 12 periods of evaluation (sub-plots) and 6 hours of evaluation (sub-subplots) and 3 blocks. The methodology was based on the principle that the $\mathrm{CO}_{2}$ released by an area of soil is absorbed by a solution of $0.5 \mathrm{~N} \mathrm{KOH}$ and determined titrimetricallyc with $\mathrm{HCl} 0.1 \mathrm{~N}$. The evolution of $\mathrm{CO}_{2}$ varied throughout the day in function of weather factors, mainly temperature. The rate of $\mathrm{CO}_{2}$ tended to release more in the afternoon. Due to the importance of soil and air temperatures in many processes that occur in soil, more studies are needed in the semiarid region of Brazil.
\end{abstract}

Keywords: Semiarid, Climate and Microbial activity.

\footnotetext{
${ }^{1}$ Recebido em 02.09.2009 e aceito para publicação em 02.05.2011.

${ }^{2}$ Universidade Federal de Alagoas, UFAL, Brasil. E-mail: <kdaraujo@yahoo.com.br>.

${ }^{3}$ Universidade Federal de Campina Grande, UFCG, Brasil. E-mail: <renilson@dca.ufcg.edu.br> .

${ }^{4}$ Instituto Nacional do SemiÁrido, INSA, Brasil. E-mail: <albericio@uol.com.br>.

${ }^{5}$ Universidade Federal do Maranhão, UFMA, Brasil. E-mail: <hnparente@hotmail.com>.
} 


\section{INTRODUÇÃO}

A atividade microbiana ou respiração edáfica é o $\mathrm{CO}_{2}$ liberado tanto pela atividade dos microrganismos quanto pelo resultante da respiração do sistema radicular dos vegetais de uma área (POGGIANI et al., 1977). É a soma total de todas as atividades metabólicas em que o $\mathrm{CO}_{2}$ é produzido, podendo este ser utilizado como índice para monitorar as respostas dos ecossistemas aos distúrbios (BEHERA et al., 1990).

Para Calgaro et al. (2008), a atividade microbiana seria a maneira indireta de avaliação do impacto ambiental, uma vez que aumento demasiado da atividade microbiana elevará a taxa de $\mathrm{CO}_{2}$ liberado para a atmosfera, favorecendo o aquecimento global ou efeito-estufa e reduzindo a camada de ozônio (TSAI et al., 1992).

Poggiani et al. (1977) verificaram que as condições climáticas e, ou, meteorológicas possuem acentuada influência sobre a evolução de $\mathrm{CO}_{2}$ do solo. Um dos fatores que contribuem para o aumento de $\mathrm{CO}_{2}$ é a elevação da temperatura do solo. Para Bley Jr. (1999), a respiração do solo eleva-se com a temperatura, e para determinada temperatura ela é maior em condições de umidade ótima.

O aumento da temperatura do solo até determinado valor eleva a cinética das conversões enzimáticas microbianas, sendo um indicador da intensidade de decomposição. Essa intensidade mostra-se distinta no curso diário e anual e depende do clima e da atividade biológica do solo (CHIMNER, 2004).

Poucos experimentos estudaram os efeitos das interações de aumentos nas concentrações de $\mathrm{CO}_{2}$ associados a aumentos da temperatura nos ecossistemas (SOUTO et al., 2009), notadamente na região semiárida do Nordeste do Brasil (ARAUJO, 2005).

Em regiões de clima temperado, os solos de floresta, charneca e sob herbáceas apresentam respiração, em média, entre 100 e $500 \mathrm{mg} \mathrm{CO}_{2} \mathrm{~m}^{-2} \mathrm{~h}^{-1} \mathrm{e}$, em campos, até $1.000 \mathrm{mg}$ de $\mathrm{CO}_{2} \mathrm{~m}^{-2} \mathrm{~h}^{-1}$. Em solos de floresta tropical ricos em material nutritivo, pode-se medir uma taxa entre 1.000 e $1.200 \mathrm{mg}$ de $\mathrm{CO}_{2} \mathrm{~m}^{-2} \mathrm{~h}^{-1}$ durante o período úmido do ano; sobre solos arenosos, regiões secas e nas tundras, somente de 50 a $200 \mathrm{mg}$ de $\mathrm{CO}_{2} \mathrm{~m}^{-2} \mathrm{~h}^{-1}$ (SINGH; GUPTA, 1977).

Estudos de cinética da respiração edáfica ajudam explicar muitos processos que ocorrem no solo e são de fundamental importância para a recuperação de áreas degradadas (SOUTO et al., 2009). A estimativa através da liberação de $\mathrm{CO}_{2}$ mostra-se indicada como uma das ferramentas para avaliar a recuperação de áreas degradadas, pelo seu baixo custo, eficiência e por indicar mudanças rapidamente (PASSIANOTO et al., 2001). Os métodos de laboratório e campo são adequados para o estudo da atividade microbiana, por respiração do solo. No entanto, o método de avaliação com medições não instantâneas da atividade respiratória no campo mostra-se importante para a complementação dos resultados. Além disso, esse método é simples, de baixo custo e apresenta maior sensibilidade, por considerar as condições naturais (ASSIS JÚNIOR et al., 2003).

O objetivo deste trabalho foi avaliar a cinética de evolução do dióxido de carbono (atividade microbiana) associada aos elementos meteorológicos em três áreas contíguas de caatinga, nas condições do semiárido paraibano.

\section{MATERIAL E MÉTODOS}

\section{1. Área de estudo}

O trabalho foi realizado na Estação Experimental Bacia Escola, pertencente à Universidade Federal da Paraíba (UFPB), localizada no Município de São João do Cariri, PB, com coordenadas 7²3'30" S e 36³1'59" W Gr, altitudes variando entre 400 e $600 \mathrm{~m}$, localizada na zona fisiográfica do Planalto da Borborema, na mesorregião da Borborema e da microrregião do Cariri Oriental. Apresenta relevo suave-ondulado sobre o embasamento Cristalino.

Na região predominam o clima Bsh quente com chuvas de verão e o bioclima 2 b com 9 a 11 meses secos, denominado subdesértico quente de tendência tropical, segundo a classificação de Gaussen. A temperatura varia de $27,2{ }^{\circ} \mathrm{C}$ no período de novembro-março a $23,1{ }^{\circ} \mathrm{C}$ em julho, com precipitações de $400 \mathrm{~mm} /$ ano e umidade relativa do ar de $70 \%$, aproximadamente.

Os solos predominantes de acordo com Chaves e Chaves (2000) são: Luvissolo Crômico vértico, raso a moderadamente profundo, com sequência de horizontes do tipo A, B e C de textura argilosa ou média, bem drenado, desenvolvido de rochas metamórficas; Vertissolo, argiloso a muito argiloso e menos desenvolvido que o Luvissolo, com sequência de A-C-R. Quando seco, apresenta fendilhamento (gretas de ressecamento) em razão de possuir argila expansiva 
e atividade alta e Neossolo Lítico, raso, com sequência de horizonte A-R, textura arenosa ou média, moderadamente drenado, com horizonte A fraco ou moderado.

A vegetação que recobre a região estudada é a Caatinga (vegetação caducifólia espinhosa), apresentando-se, em grande parte, degradada.

\subsection{Avaliação da cinética de evolução de dióxido de carbono $\left(\mathrm{CO}_{2}\right)$ por meio da atividade microbiana}

A cinética de liberação de $\mathrm{CO}_{2}$ foi realizada em três áreas contíguas de Caatinga correspondente aos três tratamentos: $\mathrm{T}_{1}$ (10 animais -1 animal/ $3.200 \mathrm{~m}^{2}$ ), $\mathrm{T}_{2}$ (5 animais -1 animal/6.400 $\mathrm{m}^{2}$ ) e $\mathrm{T}_{3}$ (Controle - Sem animais). A área do experimento totalizou 9,6 ha, e em cada tratamento foi utilizada uma área de 3,2 ha.

Foram selecionados em cada tratamento seis pontos de coleta (definidos ao acaso), para determinação da cinética de liberação de $\mathrm{CO}_{2}$ De modo que os tratamentos foram distribuídos em delineamento de blocos inteiramente casualizados e arranjados em parcela subsubdividida (3x12x6), sendo três taxas de lotação (Parcela), 12 épocas de avaliação (Subparcelas) e $6 \mathrm{~h}$ de avaliação (Subsubparcelas) e três blocos.

As taxas de evolução de $\mathrm{CO}_{2}$ (atividade microbiana) foram realizadas a cada $2 \mathrm{~h}$ no período diurno (das 5 às 17 h), mediante a metodologia descrita por Grisi (1978), em que o $\mathrm{CO}_{2}$ liberado por uma área de solo é absorvido por uma solução de $\mathrm{KOH} 0,5 \mathrm{~N}$ e pela dosagem por titulação com $\mathrm{HCl}$ 0,1 N. Foram considerados como indicadores a fenolftaleína e o alaranjado de metila 1\% preparado segundo Morita e Assumpção (1972). Foi utilizado um frasco-controle, ou testemunha, que permaneceu hermeticamente fechado e também foi submetido ao processo de titulação. A determinação do $\mathrm{CO}_{2}$ absorvido foi realizada a partir das equações:

$$
\begin{gathered}
\mathrm{ACO}_{2}=(\mathrm{A}-\mathrm{B}) \times 2 \times 2,2 \mathrm{em} \mathrm{mg} \\
\mathrm{A}^{\prime} \mathrm{CO}_{2}=\mathrm{ACO}_{2} \times(4 / 3 \times 10.000 / \mathrm{h}+\mathrm{S}) \mathrm{em} \mathrm{mg}
\end{gathered}
$$

em que:

$$
\mathrm{A}^{\prime} \mathrm{CO}_{2}=\text { absorção de } \mathrm{CO}_{2} \text {; }
$$

$\mathrm{A}=$ diferença, em $\mathrm{mL}$, entre a $1^{\mathrm{a}}$ e a $2^{\mathrm{a}}$ viragem da coloração da amostra;
$\mathrm{B}=$ diferença, em mL, entre a $1^{\mathrm{a}} \mathrm{e} 2^{\mathrm{a}}$ viragem da coloração do controle ou testemunha;

$\mathrm{h}$ = período de permanência da amostra no solo (horas); e $\mathrm{S}$ = área de abrangência do balde.

Para efetuar a medição de $\mathrm{CO}_{2}$ foram distribuídos, em cada tratamento, 18 recipientes de vidro contendo $10 \mathrm{~mL}$ de $\mathrm{KOH}$ a $0,5 \mathrm{~N}$, totalizando 54 recipientes. Esses conjuntos foram cobertos com baldes plásticos com capacidade para $22 \mathrm{~L}$.

O balde possui formato cilíndrico, com 29,8 cm de diâmetro e 36,5 cm de altura, cobrindo uma área de solo de 697,46 cm² (Figura 1). As bordas do cilindro foram enterradas cerca de 2 a $3 \mathrm{~cm}$, para evitar as trocas gasosas diretamente com a atmosfera. Cada recipiente contendo a solução de $\mathrm{KOH} \mathrm{0,5} \mathrm{N} \mathrm{foi}$ rapidamente destampado para que fixasse o $\mathrm{CO}_{2}$ liberado do solo e coberto com o balde plástico. Após 12 h de permanência no local, os baldes foram retirados e os recipientes, rapidamente tampados e acondicionados em caixa de isopor e, em seguida, titulados. As taxas de cinética de $\mathrm{CO}_{2}$ foram feitas em seis pontos de coleta, de cada tratamento, no intervalo de $2 \mathrm{~h}$ (das 5 às $17 \mathrm{~h}$ ).

Os dados meteorológicos referentes à temperatura do solo na profundidade de $10 \mathrm{~cm}$, temperatura do ar e umidade relativa do ar foram obtidos a cada 2 h (das 5 às 17 h) a partir da Estação Meteorológica Automática instalada na área experimental. As leituras de nebulosidade foram determinadas em décimos (N/10).

Os dados de $\mathrm{CO}_{2}$ foram avaliados pela análise de regressão polinomial. Na realização da análise estatística foi empregado o Software SAEG 5.0 (SAEG, 1993).

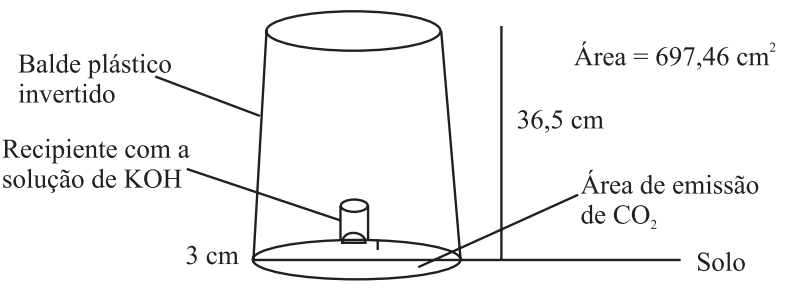

Figura 1 - Desenho esquemático do método utilizado na medição da atividade microbiana.

Figure 1 - Schematic drawing of the method used in measuring the microbial activity.

Revista Árvore, Viçosa-MG, v.35, n.5, p.1099-1106, 2011 


\section{RESULTADOS}

Constatou-se que a taxa de $\mathrm{CO}_{2}$, independentemente dos meses avaliados e dos tratamentos, apresentou variações em função das horas (Figura 2 e Tabela 1).
No tratamento I, observou-se que nos meses de janeiro, fevereiro, julho, agosto, setembro, outubro e novembro houve aumento linear de $\mathrm{CO}_{2}$ ao longo do tempo, denotando que a atividade microbiana foi
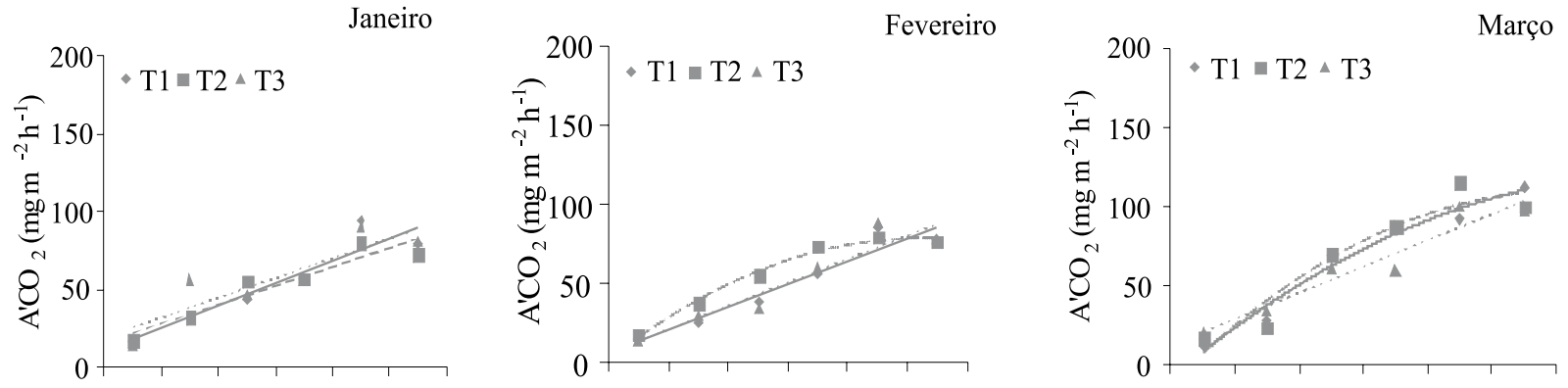

Abril

Maio

Junho
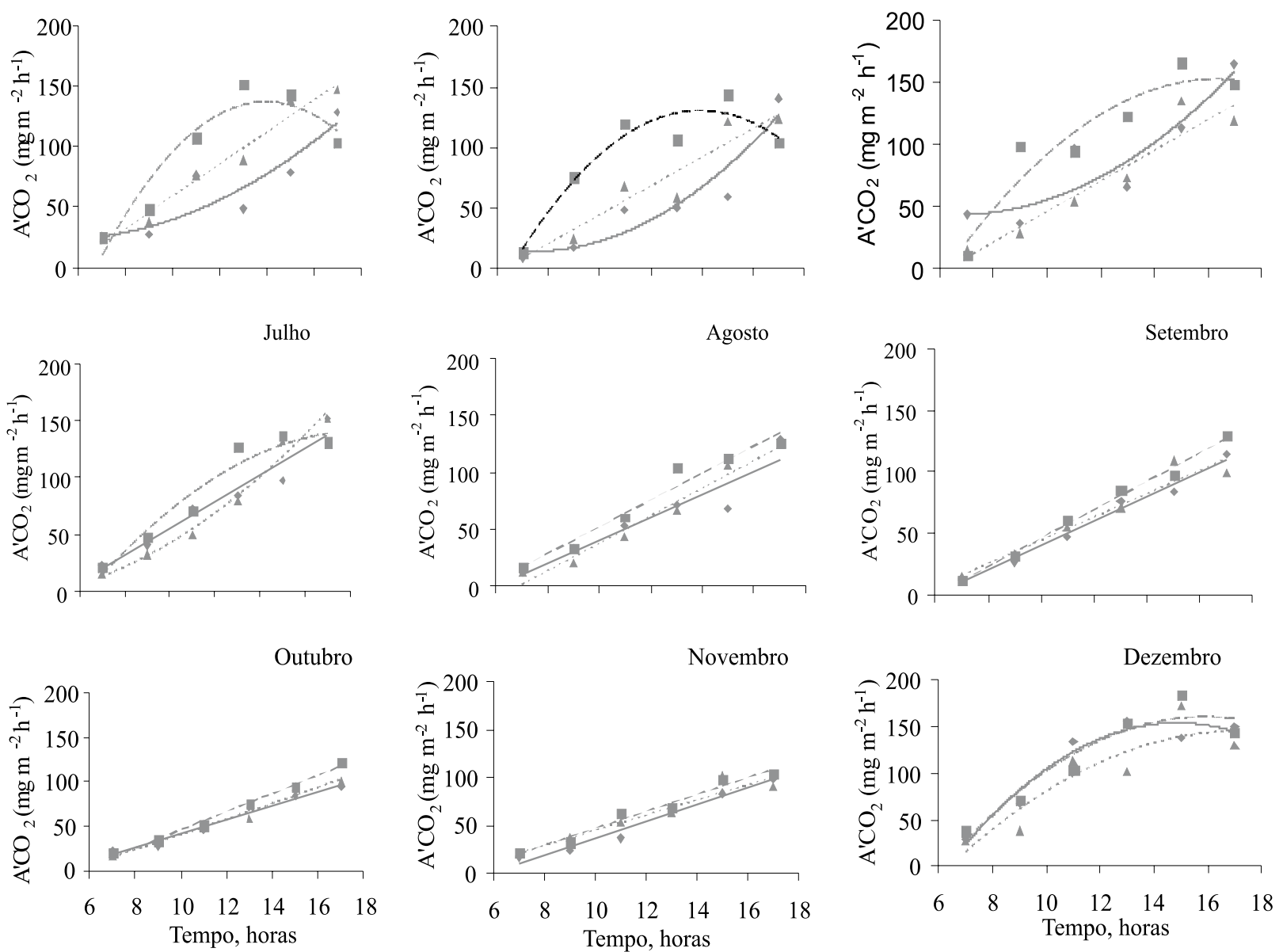

Figura 2 - Evolução do $\mathrm{CO}_{2}$ nas áreas I, II e III, no período de 12 h, em São João do Cariri, PB, ano 2008. Figure 2 - Evolution of $\mathrm{CO}_{2}$ in the areas I, II and III in the 12-hour period in São João do Cariri, PB, 2008.

Revista Árvore, Viçosa-MG, v.35, n.5, p.1099-1106, 2011 
Tabela 1 - Estimativa dos parâmetros do modelo de regressão linear da cinética de $\mathrm{CO}_{2}$ (y) em função do tempo (x), de três tratamentos.

Table 1 - Estimate of parameters of the linear regression model of the CO2 kinetics (y) against time (x) of three treatments.

\begin{tabular}{|c|c|c|c|c|c|}
\hline \multirow[t]{2}{*}{ Meses } & & \multirow{2}{*}{$\begin{array}{c}\text { Tratamentos } \\
\mathrm{B}_{2} \\
\end{array}$} & \multicolumn{3}{|c|}{ Cinética de $\mathrm{CO}_{2}(\mathrm{y})$} \\
\hline & & & $\mathrm{B}_{1}$ & $\mathrm{~B}_{0}$ & $\mathrm{R}^{2 *}$ \\
\hline \multirow[t]{3}{*}{ Janeiro } & $\mathrm{T}_{1}$ & & $7,2232 x$ & $-32,705$ & 0,88 \\
\hline & $\mathrm{T}_{2}$ & & $6,0282 x$ & $-20,001$ & 0,90 \\
\hline & $\mathrm{T}_{3}^{2}$ & & $6,372 x$ & $-19,181$ & 0,78 \\
\hline \multirow[t]{3}{*}{ Fevereiro } & $\mathrm{T}_{1}$ & & $7,3066 x$ & $-38,106$ & 0,93 \\
\hline & $\mathrm{T}_{2}$ & $-0,7051 x^{2}$ & $23,145 x$ & $-111,27$ & 0,99 \\
\hline & $\mathrm{T}_{3}^{2}$ & & $7,3767 x$ & $-37,74$ & 0,90 \\
\hline \multirow[t]{3}{*}{ Março } & $\mathrm{T}_{1}$ & $-0,557 x^{2}$ & $23,549 x$ & - 129,52 & 0,97 \\
\hline & $\mathrm{T}_{2}$ & $-0,8157 x^{2}$ & $29,637 x$ & $-160,13$ & 0,92 \\
\hline & $\mathrm{T}_{3}$ & & $8,2813 x$ & - 37,73 & 0,92 \\
\hline \multirow[t]{3}{*}{ Abril } & $\mathrm{T}_{1}^{3}$ & $0,6717 x^{2}$ & $-6,7425 x$ & 40,05 & 0,82 \\
\hline & $\mathrm{T}_{2}$ & $-2,6088 x^{2}$ & $72,881 x$ & $-372,02$ & 0,90 \\
\hline & $\mathrm{T}_{3}$ & & $13,235 x$ & - 73,261 & 0,97 \\
\hline \multirow[t]{3}{*}{ Maio } & $\mathrm{T}_{1}^{3}$ & $1,2183 x^{2}$ & $-18,018 x$ & 80,847 & 0,89 \\
\hline & $\mathrm{T}_{2}$ & $-2,3897 x^{2}$ & $66,549 x$ & - 332,7 & 0,92 \\
\hline & $\mathrm{T}_{3}$ & & $11,903 x$ & $-74,608$ & 0,91 \\
\hline \multirow[t]{3}{*}{ Junho } & $\mathrm{T}_{1}$ & $1,0796 x^{2}$ & $-14,458 x$ & 92,007 & 0,85 \\
\hline & $\mathrm{T}_{2}$ & $-1,4854 x^{2}$ & $48,686 x$ & $-246,39$ & 0,90 \\
\hline & $\mathrm{T}_{3}$ & & $12,252 x$ & $-76,438$ & 0,90 \\
\hline \multirow[t]{3}{*}{ Julho } & $\mathrm{T}_{1}$ & & $11,896 x$ & $-64,403$ & 0,94 \\
\hline & $\mathrm{T}_{2}$ & $-0,9383 x^{2}$ & $35,034 x$ & $-185,35$ & 0,94 \\
\hline & $\mathrm{T}_{3}$ & $0,6749 x^{2}$ & $-1,6372 x$ & - 8,6157 & 0,98 \\
\hline \multirow[t]{3}{*}{ Agosto } & $\mathrm{T}_{1}$ & & $10,124 \mathrm{x}$ & $-60,386$ & 0,89 \\
\hline & $\mathrm{T}_{2}$ & & $11,794 x$ & $-65,771$ & 0,96 \\
\hline & $\mathrm{T}_{3}$ & & $12,097 x$ & $-83,24$ & 0,97 \\
\hline \multirow[t]{3}{*}{ Setembro } & $\mathrm{T}_{1}^{3}$ & & $10,077 x$ & $-60,682$ & 0,98 \\
\hline & $\mathrm{T}_{2}$ & & $11,551 x$ & - 69,195 & 0,99 \\
\hline & $\mathrm{T}_{3}^{2}$ & & $9,4829 x$ & $-50,281$ & 0,93 \\
\hline \multirow[t]{3}{*}{ Outubro } & $\mathrm{T}_{1}$ & & $7,9659 x$ & - 37,937 & 0,97 \\
\hline & $\mathrm{T}_{2}$ & & $10,254 x$ & $-56,691$ & 0,99 \\
\hline & $\mathrm{T}_{3}$ & $0,0522 x^{2}$ & $7,5336 x$ & - 39,953 & 0,97 \\
\hline \multirow[t]{3}{*}{ Novembro } & $\mathrm{T}_{1}$ & & $8,8704 x$ & $-51,634$ & 0,97 \\
\hline & $\mathrm{T}_{2}$ & & $8,802 x$ & $-40,864$ & 0,97 \\
\hline & $\mathrm{T}_{3}$ & & $7,8674 x$ & $-33,465$ & 0,91 \\
\hline \multirow[t]{3}{*}{ Dezembro } & $\mathrm{T}_{1}^{3}$ & $-2,0956 x^{2}$ & $62,257 x$ & $-308,92$ & 0,95 \\
\hline & $\mathrm{T}_{2}$ & $-1,6663 x^{2}$ & $52,971 x$ & - 261,13 & 0,91 \\
\hline & $\mathrm{T}_{3}^{2}$ & $-1,2413 \times 2$ & $42,675 x$ & $-222,06$ & 0,82 \\
\hline
\end{tabular}

* Diferenciam estatisticamente a $\mathrm{p}<0,05$.

aumentando à medida que as horas foram passando, com maior incremento às $17 \mathrm{~h}$.

No tratamento II, a liberação de $\mathrm{CO}_{2}$ aumentou de forma linear nos meses de janeiro, agosto, setembro, outubro e novembro, aumentando o desprendimento com o decorrer das horas. Nos demais meses, verificou-se a tendência à redução no desprendimento de $\mathrm{CO}_{2}$ a partir das $14 \mathrm{~h}$.
No tratamento III, com exceção de julho e dezembro, nos demais meses houve efeito linear com desprendimento de $\mathrm{CO}_{2}$ com o decorrer das horas de avaliação. No mês de julho, apesar de os dados referentes à liberação de $\mathrm{CO}_{2}$ ter-se ajustado ao modelo de regressão polinomial, verificou-se que a máxima liberação ocorreu às $17 \mathrm{~h}$, semelhante ao mês de dezembro, que pela derivada da equação de regressão se constatou haver maior 
liberação desse gás no final do período de avaliação (Figura 2 e Tabela 1).

A Figura 3AD refere-se às médias das 12 observações, referentes à evolução média horária do $\mathrm{CO}_{2}$ em função da temperatura do ar e da temperatura do solo, umidade relativa do ar e nebulosidade.

Observou-se que houve flutuações nas emissões de $\mathrm{CO}_{2}$ nos diferentes horários, nos três tratamentos com menor atividade microbiana às 7 h, com 19,53;
19,43; e $17,17 \mathrm{mg} \mathrm{m}^{-2} \mathrm{~h}^{-1}$, respectivamente, quando a temperatura do ar $\left(21,2^{\circ} \mathrm{C}\right)$ e a temperatura do solo $\left(27^{\circ} \mathrm{C}\right)$ foram reduzidas e a umidade relativa do ar $(81,8 \%)$ e a nebulosidade (7/10), elevadas. As maiores liberações foram constatadas às $17 \mathrm{~h}$ (123,06 $\left.\mathrm{mg} \mathrm{m}^{-2} \mathrm{~h}^{-1}\right)$ na área I, quando a temperatura do ar e a temperatura do solo foram de 27,08 e $34,08^{\circ} \mathrm{C}$, umidade relativa do ar de 55,6\% e nebulosidade de 4/10, e às $15 \mathrm{~h}$ nas áreas II $\left(114,59 \mathrm{mg} \mathrm{m}^{-2} \mathrm{~h}^{-1}\right)$ e III $\left(120,66 \mathrm{mg} \mathrm{m}^{-2} \mathrm{~h}^{-1}\right)$, quando a temperatura do

A
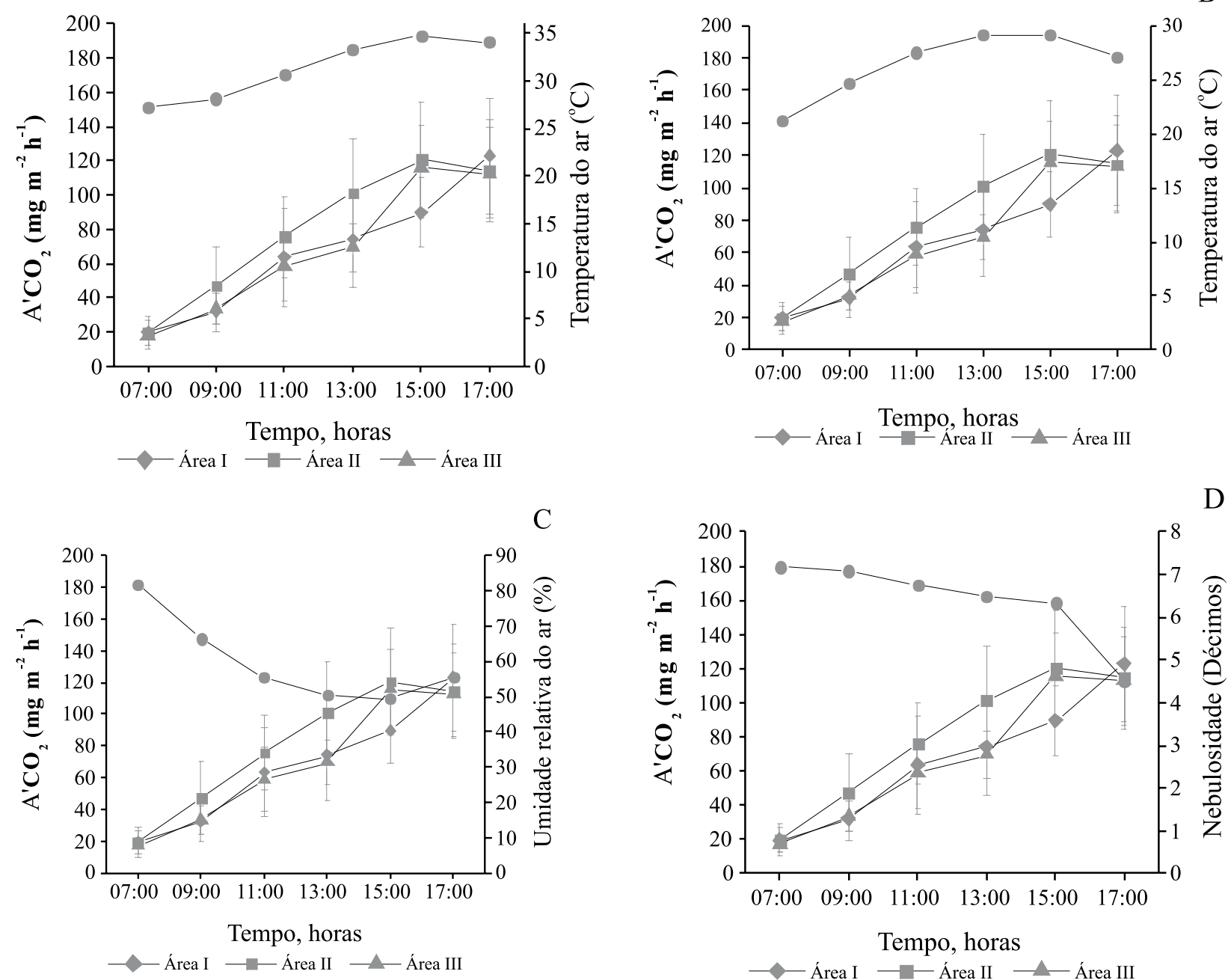

$\mathrm{C}$

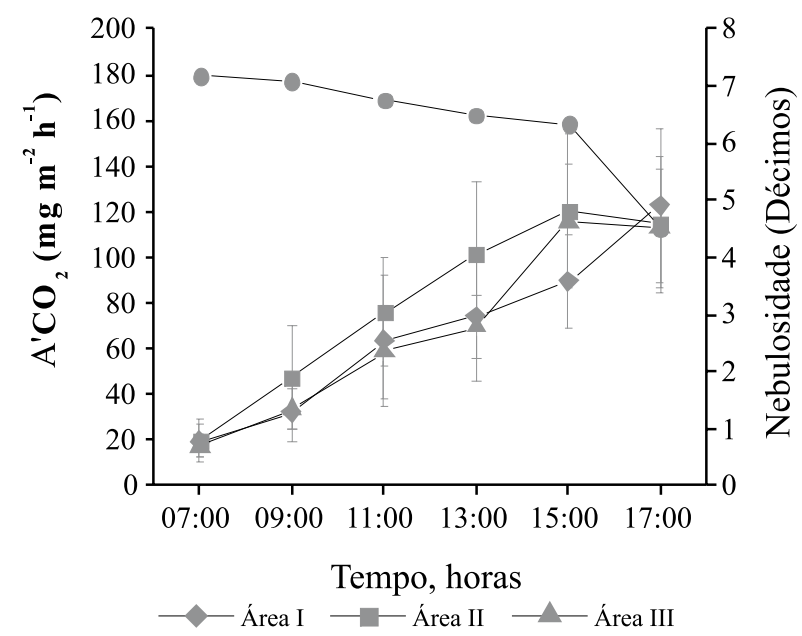

Figura 3 - Evolução média horária de $\mathrm{CO}_{2}$ em função do tempo e da temperatura do ar (A), temperatura do solo (B), umidade relativa do ar (C) e nebulosidade (D), nas áreas I, II e III, das 7 às 17 h, em São João do Cariri, PB, ano 2008. As barras verticais representam o desvio da média.

Figure 3 - Average hourly trends of $\mathrm{CO}_{2}$ in function of time and air temperature (A), soil temperature (B), air relative humidity (C) and cloudiness (D) in areas I, II and III, from 7a.m. 5 p.m. in São João do Cariri, PB, 2008. Vertical bars represent the deviation of the mean.

Revista Árvore, Viçosa-MG, v.35, n.5, p.1099-1106, 2011 
ar e a temperatura do solo foram de 29,2 e $34,8^{\circ} \mathrm{C}$ e a umidade relativa do ar de $49,4 \%$ e nebulosidade de $6 / 10$

\section{DISCUSSÃO}

Essas variações nas emissões de $\mathrm{CO}_{2}$ decorreram de variações no conteúdo de água do solo e da temperatura, com tendência a maiores perdas nos horários mais quentes. Isso é devido ao fato de a maioria dos microrganismos do solo se adaptar bem a temperaturas mais elevadas (TREVISAN et al., 2002), característica peculiar da região semiárida. De acordo com Souto et al. (2009), há indicativo de que os microrganismos aumentam sua atividade entre 40 e $45^{\circ} \mathrm{C}$ na região semiárida, e quando alcançam valores próximos aos $50{ }^{\circ} \mathrm{C}$ há inibição da atividade microbiana e, consequentemente, menor produção de $\mathrm{CO}_{2}$.

De acordo com Paul e Clark (1996), a menor atividade dos microrganismos, resultando em decréscimos de $\mathrm{CO}_{2}$ liberado em função de baixas temperaturas, está associada à adaptabilidade dos microrganismos a diferentes extremos de temperatura. Desse modo, a estabilização do $\mathrm{CO}_{2}$ nas horas de temperaturas extremas possivelmente tenha resultado de inibição da atividade microbiana, sendo uma resposta dos microrganismos às condições reinantes do ambiente.

Já nas outras regiões geográficas, como Eldorado do Sul (RS), Cattelan e Vidor (1990) mencionaram haver tendência geral de estímulo da biomassa em épocas de boa disponibilidade hídrica e de temperaturas amenas. Nas épocas secas e de temperaturas altas, assim como naquelas muito frias, o efeito é negativo.

De forma semelhante, Bley Jr. (1999), em estudo realizado na Região Sul do Brasil, citou que os microrganismos do solo não resistem mais que algumas horas à temperatura acima de $40^{\circ} \mathrm{C}$. A morte desses organismos ou a paralisação de sua atividade interrompe os ciclos de transformação de minerais em nutrientes para as plantas, com evidentes prejuízos às culturas. Assim, em face da importância da temperatura nos diversos processos que ocorrem no solo, são necessários mais estudos no semiárido brasileiro (SOUTO et al., 2009).

Houve tendência de maiores perdas nos horários mais quentes e nos locais onde a penetração dos raios solares foi facilitada pela presença de clareiras ou entre as ilhas vegetais, conforme ocorreu na área II, que é a mais antropizada das três áreas. Nesse caso, a ausência de cobertura do solo, ao permitir a incidência direta de raios solares, aumenta a temperatura do solo, intensificando a atividade microbiana, demonstrada pelo aumento da emissão de $\mathrm{CO}_{2}$ do solo para a atmosfera (COSTA et al., 2008).

Assim, pequenas alterações na MOS, que é muito sensível à temperatura, podem acelerar os seus processos de mineralização e resultar na liberação de grandes quantidades de $\mathrm{CO}_{2}$ para a atmosfera, elevando a concentração dos gases do efeito-estufa e, consequentemente, contribuindo para o aquecimento global (JENKINSON et al., 1991; LISBOA et al., 2006).

\section{CONCLUSÕES}

- A evolução de $\mathrm{CO}_{2}$ variou ao longo do dia em razão dos elementos meteorológicos, principalmente temperatura.

- A taxa de $\mathrm{CO}_{2}$ apresentou tendência à maior liberação no final da tarde.

- Em decorrência da importância da temperatura do solo e do ar nos inúmeros processos que ocorrem no solo, são necessários mais estudos na região semiárida do Brasil.

\section{REFERÊNCIAS}

\section{ARAUJO, K. D. Variabilidade temporal das condições climáticas sobre as perdas de $\mathrm{CO}_{2}$ na encosta do açude Namorados, em São João do Cariri-} PB. 2005. 101f. Dissertação (Mestrado em Manejo e Conservação do Solo e Água) - Centro de Ciências Agrárias, Universidade Federal da Paraíba, Areia, 2005.

ASSIS JÚNIOR, S. L. et al. Atividade microbiana do solo em sistemas agroflorestais, monoculturas, mata natural e área desmatada. Revista

Árvore, v.27, n.1, p.35-41, 2003.

BEHERA, N.; JOSHI, S. K.; PATI, D. P. Root contribution to total soil metabolism in a forest soil from Orissa, Índia. Forest Ecology and Management, v.36, n.1, p.125-134, 1990.

BLEY JR., C. Erosão Solar: riscos para a agricultura nos trópicos. Ciência Hoje, v.25, n.148, p.24-29, 1999. 
CALGARO, H. F. et al. Resíduos orgânicos como recondicionante de subsolo degradado e efeitos na atividade microbiana e fertilidade em cultivo de barbatimão. Revista Árvore, v.32, n.6, p.1069-1079, 2008.

CATTELAN, A. J.; VIDOR, C. Flutuações na biomassa, atividade e população microbiana do solo, em função de variações ambientais.

Revista Brasileira de Ciência do Solo, v.14, n.1, p.133-142, 1990.

CHAVES, L. H. G.; CHAVES, I. B.; VASCONCELOS, A. C. F. Salinidade das águas superficiais e suas relações com a natureza dos solos na Bacia Escola do açude Namorados. Campina Grande: BNB/UFPB, 2000. 54p. (Boletim Técnico).

CHIMNER, R. A. Soil respiration rates of tropical peatlands in Micronesia and Hawaii. Wetlands, v.24, n.1, p.51-56, 2004.

COSTA, F. S. et al. Estoque de carbono orgânico no solo e emissões de dióxido de carbono influenciadas por sistemas de manejo no Sul do Brasil. Revista Brasileira de Ciência do Solo, v.32, n.1, p.323-332, 2008.

GRISI, B. M. Método químico de medição de respiração edáfica: alguns aspectos técnicos. Ciência e Cultura, v.30, n.1, p.82-88, 1978.

JENKINSON, D. S.; ADAMS, D. E.; WILD, A. Model estimates of $\mathrm{CO}_{2}$ emissions from soil in response to global warming. Nature, v.351, p.304-306, 1991.

LISBOA, C. C. et al. Emissões de $\mathrm{CO}_{2}$ de solos submetidos a um aumento de temperatura. In: XVI REUNIÃO BRASILEIRA DE MANEJO E CONSERVAÇÃO DO SOLO E DA ÁGUA, 2006, Aracaju. Anais... Aracaju: Sociedade Brasileira de Ciência do Solo, 2006. CD ROM.
MORITA, T.; ASSUNPÇÃO, R. M. V. Manual de soluções, reagentes e solventes. São Paulo: Edgard Blucher, 1972. p.629.

PASSIANOTO, C. C. et al. Atividade e biomassa microbiana no solo com a aplicação de dois diferentes lodos de curtume. Revista Brasileira de Agrociência, v.7, n 2, p.125-130, 2001.

PAUL, E. A.; CLARK, F. E. Soil microbiology and biochemistry. London: Academic Press, 1996. 340p.

POGGIANI, F. et al. Respiração edáfica em plantações de coníferas e folhosas exóticas em área de cerrado do estado de são Paulo. IPEF, n.14, p.129-148, 1977.

\section{SAEG. Sistema para análises}

estatísticas. Viçosa, MG: Fundação Arthur Bernardes, 1993. v - 5.0 (Reg.07517-5A).

SINGH, J. S.; GUPTA, S. R. Plant decomposition and soil respiration in terrestrial ecosystems. Revista de Botanica, n.43, p.449-528, 1977.

SOUTO, P. C. et al. Cinética da respiração edáfica em dois ambientes distintos no semi-árido da Paraíba, Brasil. Caatinga, v.22, n3, p.52-58, 2009.

TREVISAN, R.; MATTOS, M. L. T.; HERTER, F. G. Atividade microbiana em argissolo vermelhoamarelo distrófico típico coberto com aveia preta (Avena sp.) no outono, em um pomar de pessegueiro. Científica Rural, v.7, n.2, p.83-89. 2002.

TSAI, S. M.; CARDOSO, E. J. B. N.; NEVES, M. C. P. Microbiologia do solo. Campinas: Sociedade Brasileira de Ciência do Solo, 1992. 360p. 